\title{
BMJ Open Effect of faecal calprotectin testing on referrals for children with chronic gastrointestinal symptoms in primary care: study protocol for a cluster randomised controlled trial
}

\author{
Sophie Ansems (D) , ${ }^{1}$ Marjolein Berger, ${ }^{1}$ Patrick Ferry van Rheenen, ${ }^{2}$ \\ Karin Vermeulen, ${ }^{3}$ Gina Beugel, ${ }^{1}$ Maria Couwenberg, ${ }^{1}$ Gea Holtman (1) ${ }^{1}$
}

To cite: Ansems S, Berger M, van Rheenen PF, et al. Effect of faecal calprotectin testing on referrals for children with chronic gastrointestinal symptoms in primary care: study protocol for a cluster randomised controlled trial. BMJ Open 2021;11:e045444. doi:10.1136/ bmjopen-2020-045444

- Prepublication history and additional supplemental material for this paper are available online. To view these files, please visit the journal online (http://dx.doi.org/10.1136/ bmjopen-2020-045444)

Received 02 0ctober 2020 Accepted 07 July 2021

\section{Check for updates}

(c) Author(s) (or their employer(s)) 2021. Re-use permitted under CC BY-NC. No commercial re-use. See rights and permissions. Published by BMJ.

${ }^{1}$ General Practice and Elderly Care Medicine, University Medical Centre Groningen, Groningen, The Netherlands

${ }^{2}$ Paediatric Gastroenterology, University Medical Centre Groningen Beatrix Childrens Hospital, Groningen, The Netherlands

${ }^{3}$ Epidemiology, University Medical Centre Groningen, Groningen, The Netherlands

Correspondence to

Sophie Ansems;

s.m.ansems@umcg.n

\section{ABSTRACT}

Introduction Children with chronic gastrointestinal symptoms are frequently seen in primary care, yet general practitioners (GPs) often experience challenges distinguishing functional gastrointestinal disorders (FGID) from organic disorders. We, therefore, aim to evaluate whether a test strategy that includes point-of-care testing (POCT) for faecal calprotectin (FCal) can reduce the referral rate to paediatric specialist care among children with chronic gastrointestinal symptoms. The study findings will contribute to improving the recommendations on FCal use among children in primary care.

Methods and analysis In this pragmatic cluster randomised controlled trial, we will randomise general practices into intervention and control groups. The intervention group will use FCal-POCT when indicated, after completing online training about its indication, interpretation and follow-up as well as communicating an FGID diagnosis. The control group will test and treat according to Dutch GP guidelines, which advise against FCal testing in children. GPs will include children aged 4-18 years presenting to primary care with chronic diarrhoea and/or recurrent abdominal pain. The primary outcome will be the referral rate for children with chronic gastrointestinal symptoms within 6 months after the initial assessment. Secondary outcomes will be evaluated by questionnaires completed at baseline and at 3- and 6-month follow-up. These outcomes will include parental satisfaction and concerns, gastrointestinal symptoms, impact of symptoms on daily function, quality of life, proportion of children with paediatrician-diagnosed FGID referred to secondary care, health service use and healthcare costs. A sample size calculation indicates that we need to recruit $158 \mathrm{GP}$ practices to recruit 406 children.

Ethics and dissemination The Medical Research Ethics Committee (MREC) of the University Medical Center Groningen (The Netherlands) approved this study (MREC number: 201900309). The study results will be made available to patients, GPs, paediatricians and laboratories via peer-reviewed publications and in presentations at (inter)national conferences.
Strengths and limitations of this study

- Faecal calprotectin (FCal) has high diagnostic value in children in primary care, but it is not known if its use delivers sufficient benefits to patients or if its cost justifies routine use.

- To facilitate optimal FCal use, we believe that training about the indication, interpretation, follow-up and communication of FCal results will be key to introducing this point-of-care test.

- The study results can be directly translated to daily practice in primary care because of its pragmatic design and the incorporation of the test strategy in routine clinical practice.

- Due to this pragmatic design, GPs will not be blinded to either group allocation or study outcomes.

- The cluster design means that we must be aware of the risk of selection bias.

Trial registration number The Netherlands Trial Register: NL7690 (Pre-results)

\section{INTRODUCTION}

Background and rationale

A Dutch general practitioner (GP) typically sees approximately 10 children with chronic gastrointestinal symptoms each year. ${ }^{12}$ At least $90 \%$ of these children will have functional gastrointestinal disorders (FGID). ${ }^{3}$ However, before this diagnosis can be made, the GP should ascertain after appropriate medical evaluation that the symptoms cannot be attributed to inflammatory bowel disease (IBD), coeliac disease and other causes. ${ }^{4}$ However, it is a diagnostic challenge to differentiate between FGID and these organic diseases because their clinical presentations can be very similar. Referring and testing children to identify these low-prevalent disorders then delays appropriate treatment for FGID 
and can lead to unnecessary suffering. ${ }^{56}$ Additionally, we want to prevent specialists' time taken up with FGID, as it is considered a complex and time-consuming problem in specialist care. ${ }^{1}$ At the same time, it is critical that we avoid delaying the diagnosis and appropriate treatment of IBD and coeliac disease to minimise complications such as anaemia and growth failure, ${ }^{7-10}$ and in the case of IBD, delayed sexual maturation, ${ }^{7}$ stricturing complications ${ }^{10}$ and internal fistulising complications. ${ }^{10-12}$

The Dutch Society of General Practitioners (Nederlands Huisartsen Genootschap; NHG) recommends testing tissue transglutaminase IgA and total serum IgA for suspected coeliac disease and testing haemoglobin, leukocytes and erythrocyte sedimentation rate (ESR) for suspected IBD. ${ }^{13}$ However, these blood tests cannot exclude IBD, having a sensitivity of only $0.43-0.57,{ }^{14-16}$ and they are invasive and potentially traumatic for children. ${ }^{17}$ By contrast, faecal calprotectin (FCal) is a noninvasive marker of intestinal inflammation that has been shown in recent observational studies to exclude IBD safely in children with chronic gastrointestinal symptoms and additional alarm symptoms in primary care settings (sensitivity, $0.99-1.00 ; 95 \%$ CI 0.81 to 1.00$).{ }^{18-20}$ When tested in children without alarm symptoms, however, the positive predictive value decreases due to the low prevalence $(<1 \%)$ of IBD in this population. ${ }^{21}$ The number of children referred for further diagnostic evaluation may, therefore, increase unintentionally. However, we emphasise that FCal is especially appropriate for use in primary care due to its high negative predictive value (1.00; $95 \%$ CI 0.94 to 1.00 ) rather than its positive predictive value $(0.59 ; 95 \%$ CI 0.41 to 0.75$) .{ }^{18}$

The last decade has seen an increasing focus on pointof-care testing (POCT) in primary care to improve rapid decision-making and triage at the time and place of patient care. ${ }^{22-24}$ FCal is available as a POCT for which results are available the same day and samples do not need to be sent to a laboratory, yet it retains characteristics that are comparable to the standard laboratory test. $^{25-27}$ Therefore, the FCal-POCT could decrease a GPs diagnostic uncertainty and provide early reassurance for both parents and children that a potential harmful disease (IBD) can be safely excluded. To optimise FCalPOCT implementation, proper training is needed about its indication, interpretation and follow-up. ${ }^{22}$ Perceived parental pressure for a referral is another relevant factor that may influence the decision to refer children with chronic gastrointestinal symptoms. Therefore, GPs must also receive communication skills training to explain the results, the pros and cons of referral, the natural course of (functional) symptoms and when to consult again. ${ }^{28}$

\section{Hypothesis}

We hypothesise that FCal-POCT, when combined with online training about the indication, interpretation, follow-up of testing and communicating an FGID diagnosis, will increase patient satisfaction and substantially reduce the referral rate for children with chronic gastrointestinal symptoms from primary to secondary care, as compared with usual care.

\section{METHODS AND ANALYSIS}

This protocol is reported in accordance with the Standard Protocol Items: Recommendations for Interventional Trials guidelines ${ }^{29}$ and the extended Consolidated Standards of Reporting Trials statement for cluster trials. ${ }^{30}$

\section{Design and setting}

This is a pragmatic clustered randomised controlled trial with 1:1 randomisation, at the level of the GP practice, to either an intervention group or a control group (see figure 1). From October 2019 to October 2020, GP practices in the Netherlands were invited to participate in the study. The Netherlands has a primary healthcare system in which the GP functions as the gatekeeper to specialist (ie, paediatric) care, comparable to the systems in among others Canada and the UK.

The primary outcome (referral to paediatric specialist care) will be assessed at an individual level within 6 months after baseline GP consultation, defined as the first consultation at which a child meets the criteria for inclusion. The first child was included on 15 October 2019 and the inclusion of children will end once the required sample size is reached (planned October 2021). Six months later is the planned study end date (April 2022).

\section{Study population}

Every general practice in the Netherlands is eligible for participation in our study, including all GPs and GP trainees working at those practices. GPs will be asked to include children meeting the following criteria: age 4-18 years; with chronic diarrhoea (defined as soft to watery stool for $\geq 2$ weeks or $\geq 2$ episodes in the past 2 months) and/or with recurrent or chronic abdominal pain (defined as abdominal pain with a recurrent character for $\geq 2$ months or $\geq 2$ episodes in the past 2 months). Children will be excluded if they have a history of chronic organic gastrointestinal disease (eg, coeliac disease or IBD) or if they have had an endoscopic evaluation, referral to paediatric care for gastrointestinal symptoms or an FCal result within the preceding 6 months.

\section{Intervention and control group}

Randomisation and blinding

GP practices will be randomised by a computer-generated list using varying block randomisation in 1:1 ratio by an independent researcher $(\mathrm{H}$ van der Worp, $\mathrm{PhD}$ ) not involved in the project. To reduce the risk of contamination, all GPs working at a given GP practice will be allocated as a cluster in the same study arm. On the rare occasion that a GP works in multiple practices not allocated to the same study arm, this GP will only include children in the practice that first participated in this study. GPs, children and parents will not be blinded to 


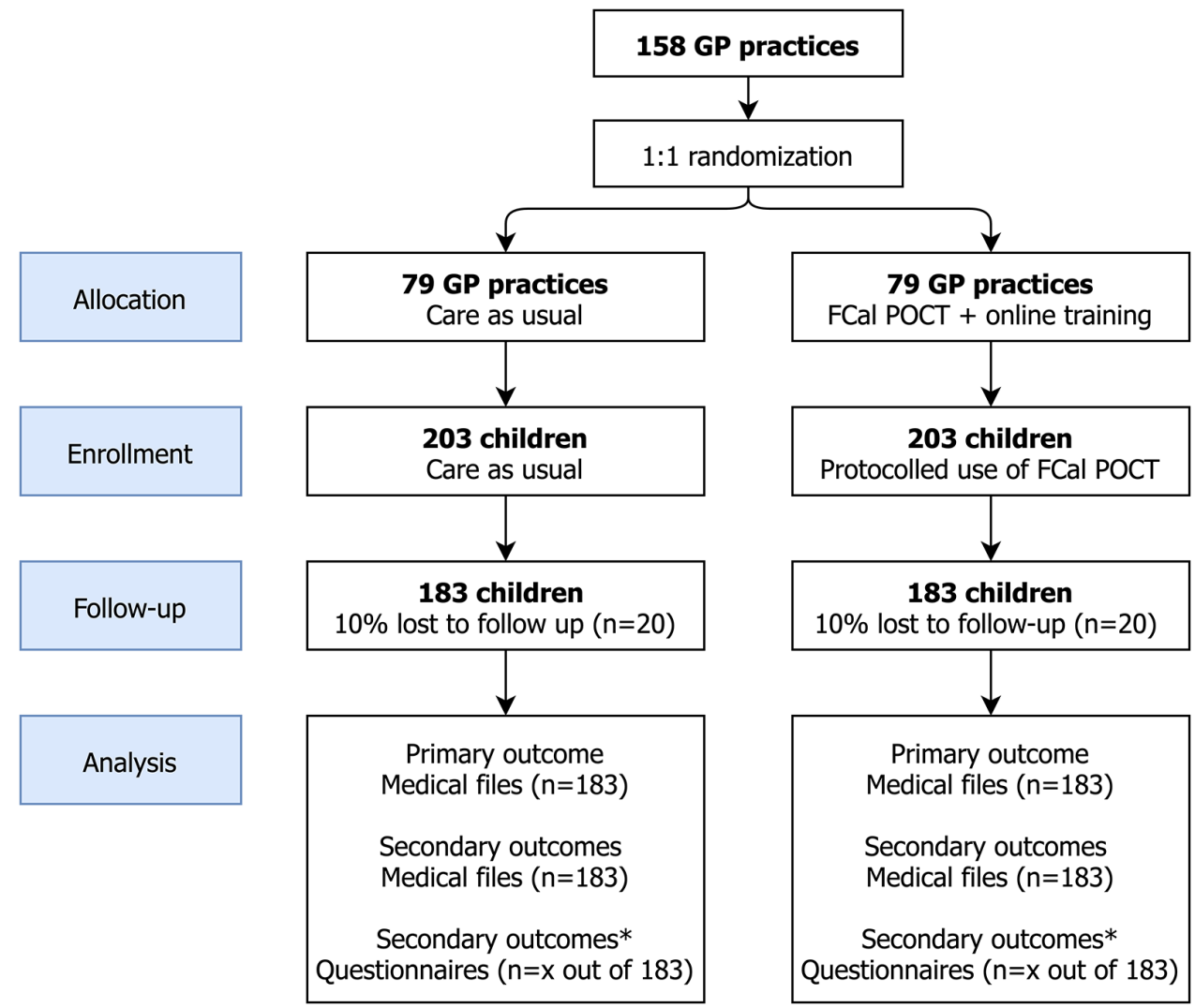

Figure 1 Study design. * Secondary outcomes evaluated by questionnaires will only be assessed in children who provide informed consent. We estimate that $50 \%$ of the recruited children will provide informed consent. FCal, faecal calprotectin; GP, general practitioner; POCT, point-of-care testing.

the intervention, but the research team will be blinded to study group assignment for the statistical analysis.

Control group: care as usual

GPs in the control group will provide care as usual according to the NHG guidelines, which recommends not using FCal testing in children. ${ }^{13}$ 31-33 It will nevertheless still be possible for them to request laboratory FCal testing or to refer the child for further diagnostic testing, if deemed necessary. All GPs will receive an information leaflet about what is considered care as usual per the NHG guideline (online supplemental file 1).

Intervention group: FCal-POCT plus online training

FCal-POCT devices will be made available to GPs for use in their practices. All participating GPs will complete the obligatory online training and will receive the same information leaflet as the control group. However, this leaflet will be amended to recommend FCal instead of ESR, leucocytes and haemoglobin when IBD is suspected. Although

Table 1 Definitions of alarm symptoms for IBD

\begin{tabular}{lll}
\hline Alarm symptom & Method of ascertainment & Definition of positive finding \\
\hline Positive family history for IBD & History & Affected first-degree relative(s) \\
\hline Rectal bleeding & History & \\
Involuntary weight loss & History +physical examination & \\
Decreased growth velocity & History +physical examination & \\
Aphthous stomatitis & History +physical examination & \\
Arthritis & History +physical examination & \\
Eye inflammation & History +physical examination & Uveitis, (epi) scleritis \\
\hline Skin abnormalities & Physical examination & Pyoderma gangrenosum, psoriasis, erythema nodosum \\
\hline Perianal abnormalities & Physical examination & $\begin{array}{l}\text { Skin tags, perianal fistulas, haemorrhoids, perianal fissures, perianal } \\
\text { abscesses }\end{array}$ \\
\hline
\end{tabular}

These definitions apply to the alarm symptoms mentioned in the protocol, figures and online supplemental files.

$\mathrm{IBD}$, inflammatory bowel disease. 
GPs are instructed to only use FCal when the child presents with alarm symptoms (table 1), FCal use will be at their own discretion. Consequently, children without FCal testing may also be included and GPs may also test and include children with alarm symptoms other than in the online training or children without alarm symptoms.

\section{The FCal-POCT: IBDoC}

The IBDoc home testing application (BÜHLMANN Laboratories AG, Schönenbuch, Switzerland) will be used. This is an in vitro diagnostic immunoassay for quantitatively determining FCal in human stool. ${ }^{34}$ Originally developed for self-testing by trained patients at home, it is also suitable for use in near-patient or laboratory settings. ${ }^{34}$ In a recent head-to-head comparison of three FCal-POCT devices in children with IBD, the IBDoc device had the best agreement with ELISA and produced significantly fewer reading errors compared with the other FCal-POCT devices. ${ }^{27}$ In the intervention group, trained research staff will teach GP assistants to use the IBDoc device during a $60 \mathrm{~min}$ face-to-face training session.

\section{Online training for GPS}

The content of the training was developed during two expert panel sessions with two academic paediatric gastroenterologists (PvR), two GPs (MB and MC), a psychologist, an educationalist, a clinical epidemiologist $(\mathrm{GAH})$ and a clinical chemist. In the first session, we formulated the FCal-POCT test strategy based on a review of the scientific literature. ${ }^{1314181927}$ In the second session, the concept of the online training was adjusted according to the four domains of Kirkpatrick's model: reaction, learning, behaviour and results. ${ }^{35}$ Subsequently, the research team developed the online training (including video recordings) in close collaboration with the expert panel. The online training was tested by five GPs (academic and nonacademic) before implementation.

The final 60 minutes online training for GPs reflects the FCal-POCT test strategy. It has been shown that an FCal value $<50 \mu \mathrm{g} / \mathrm{g}$ can safely exclude IBD in children in primary care (sensitivity of $0.99-1.00$ (95\% CI 0.81 to 1.00$)$ ). ${ }^{18}{ }^{19}$ Additionally, an FCal value $>250 \mu \mathrm{g} / \mathrm{g}$ has a specificity of $0.98(95 \%$ CI 0.92 to 0.99$).{ }^{18}$ However, an FCal value $>250 \mu \mathrm{g} / \mathrm{g}$ also has a high false-positive rate (13\%) when tested in a population of children both with and without alarm symptoms. ${ }^{18} 36$ Therefore, it is recommended to test only those children with alarm symptoms (table 1), to monitor those with an FCal value of $50-250 \mu \mathrm{g} / \mathrm{g}$ and to refer those with an FCal value $>250 \mu \mathrm{g} / \mathrm{g}$. We selected the alarm symptoms for IBD with the highest discriminatory power. We are of the opinion that adding less discriminating alarm symptoms to the training will unintentionally increase the number of false-positive findings. In addition to the indication and interpretation of cut-off values, the online training includes details on the follow-up of test results between 50 and $250 \mu \mathrm{g} / \mathrm{g}$, how to communicate the FCal result and how to educate about FGID. ${ }^{1437}$ Figure 2 shows the flowchart for the test strategy. This features prominently throughout the online training

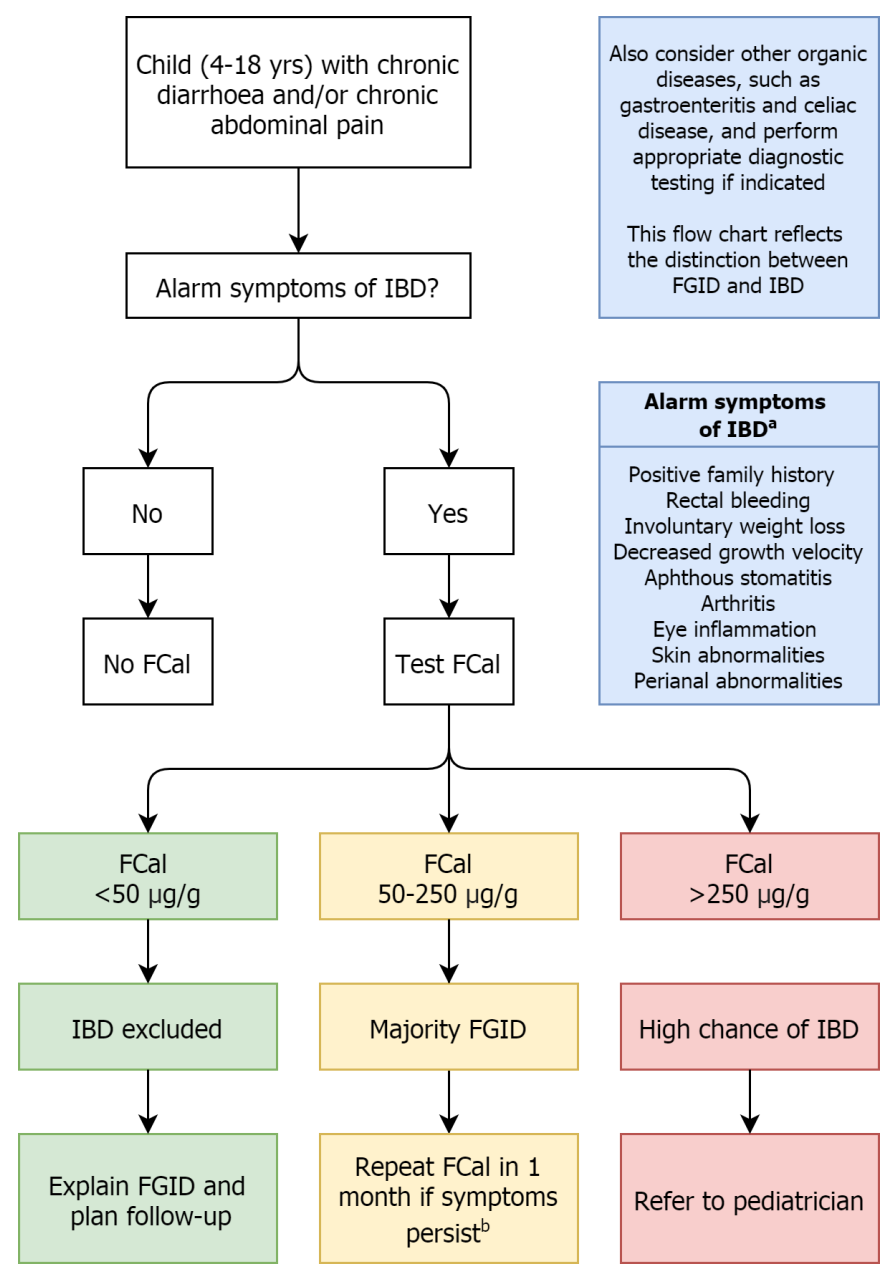

Figure 2 Test strategy in the intervention group. ${ }^{\text {a }}$ Per definitions in table 1. ${ }^{b}$ Refer to paediatrician if the repeated calprotectin after 1 month is $>50 \mu \mathrm{g} / \mathrm{g}$ to prevent diagnostic uncertainty among GPs, parents and children. FCal, faecal calprotectin; FGID, functional gastrointestinal disorder; GP, general practitioner; IBD, inflammatory bowel disease.

and is given to GPs as a desk reminder. The online training contains five modules in total: an introduction module, three modules each covering a different patient case or test scenario and a a proficiency test (online supplemental file 2). The online training uses text blocks, tables, graphs, images, videos (GP consultations with a child and parent) and interactive questions.

\section{Outcomes}

Primary outcome

The primary outcome is the proportion of referrals to secondary care within 6 months after the baseline consultation. Research staff will extract this information from the medical files of GPs.

\section{Secondary outcomes}

\section{Parental satisfaction about baseline consultation}

The Parental Medical Interview Scale measures parent satisfaction with the GP consultation. ${ }^{38}$ This questionnaire assesses physician communication with the parent and child, distress relief and adherence intent on a 
five-point Likert scale ranging from 'strongly disagree' $($ score $=1)$ to 'strongly agree' (score $=5)$. The questionnaire showed good construct validity and internal consistency $(\alpha=0.86) .^{38} 39$

\section{Parental concern at baseline and at 3 and 6 months}

At the baseline consultation and after 3 and 6 months, parents will answer the question 'How concerned do you feel about your child's gastrointestinal symptoms?' on a numeric version of a visual analogue scale (scored 1 to 10 , with 1 defined as 'not concerned' and 10 defined as 'extremely concerned'). At the baseline consultation, parents will complete an additional questionnaire about their concerns. This will cover if and where parents sought advice before contacting their physician, what their current concerns are and how the physician could provide reassurance to both the parent and child. ${ }^{40}$

\section{Self-reported gastrointestinal symptoms at baseline and at 3 and 6 months}

Self-reported gastrointestinal symptoms will be evaluated using a 10-item questionnaire that we have previously used in a study of the diagnostic value of FCal for IBD in primary care. ${ }^{41}$ This questionnaire assesses the presence of alarm symptoms as well as the duration and severity of abdominal pain and/or diarrhoea.

\section{Impact of gastrointestinal symptoms on the child's daily function at} baseline and at 3 and 6 months

The impact of symptoms on daily function will be evaluated with the Functional Disability Inventory (FDI). ${ }^{42}$ This assesses self-reported difficulty in physical and psychosocial functioning due to physical health over the past 2 weeks. Responses to 15 items are scored on 5-point scales that range from 'no trouble' (0) to 'impossible' (4). ${ }^{4}$ Items are averaged to give a composite score. Cronbach's alpha coefficient for the FDI is reported to be $0.90 .{ }^{42}$

\section{Child's quality of life at baseline and at 3 and 6 months}

Quality of life will be evaluated with the EuroQol Youth (EQ-5D-Y), a generic measure for quality of life. This instrument includes five domains (ie, mobility, self-care, usual activities, pain and discomfort and anxiety and depression) with three levels of severity (ie, no problems, some problems and a lot of problems). ${ }^{43}$ The questionnaire is feasible for use by children. ${ }^{44}$

\section{The proportion of children referred to paediatric care with FGID over 6 months}

The proportion of children diagnosed with FGID by the paediatrician will be recorded among those referred to paediatric care. This information will be extracted from the child's medical records based on letters sent by the paediatrician to the GP.

\section{Healthcare use over 6 months}

For all children, we will collect the following data from medical records: diagnostic tests, referrals to healthcare providers other than a paediatrician, medication prescriptions, GP consultation frequency and healthcare use at hospital (online supplemental file 3). For FCal in specific, we will also collect whether children return their stool samples.

\section{Costs over 6 months}

Units of medical consumption will be extracted from medical records for all children (see healthcare use). In addition, cost questionnaires will be completed by parents at baseline and at 3-month and 6-month follow-up. These will measure additional healthcare use, out-ofpocket expenses and productivity losses (absence from work) based on adapted versions of the iMTA Medical Consumption Questionnaire and the iMTA Productivity Cost Questionnaire. ${ }^{45}$

\section{Recruitment}

We will invite all GP practices connected to the Academic General Practitioner Development Network (AHON; Academisch Huisarts Ontwikkel Netwerk) via an informational letter. This network comprises 473 urban and rural GP practices in the four northern provinces of the Netherlands, and it seeks to facilitate collaboration in research, education and innovation in general practice. We will also approach GP practices throughout the Netherlands with which our research staff are connected.

GPs will identify and recruit consecutive eligible children during baseline consultations for 1 year (figure 3). Additionally, research staff will retrospectively search for eligible children seen in practice in the previous 3 months. They will search in GP registration databases using a search strategy based on International Classification of Primary Care codes (online supplemental file 4). All included children and/or parents (regardless of the recruitment strategy) will receive a patient information letter and will be asked to provide informed consent for completing questionnaires (online supplemental file 5). Consequently, secondary outcomes assessed with questionnaires will only be evaluated in children who provide this consent.

\section{Data collection}

For each eligible child, independent of inclusion during or after consultation, the GP will complete a trial inclusion form detailing the inclusion/exclusion criteria, gender, date of birth, presence of alarm symptoms and use of FCal-POCT (the latter only in the intervention group). The trial inclusion form will be sent to the researchers, and for all included children, data will be retrieved from their medical files for each consultation (including baseline) over a 6-month follow-up period in a standardised online data entry form by the research team (online supplemental file 3). Children and/or parents who provide informed consent will also complete digital questionnaires via RedCap after consultations at baseline, 3 months and 6 months. The estimated time to complete each questionnaire is $15-20 \mathrm{~min}$, and if they are not completed, the child and/or parents will automatically 


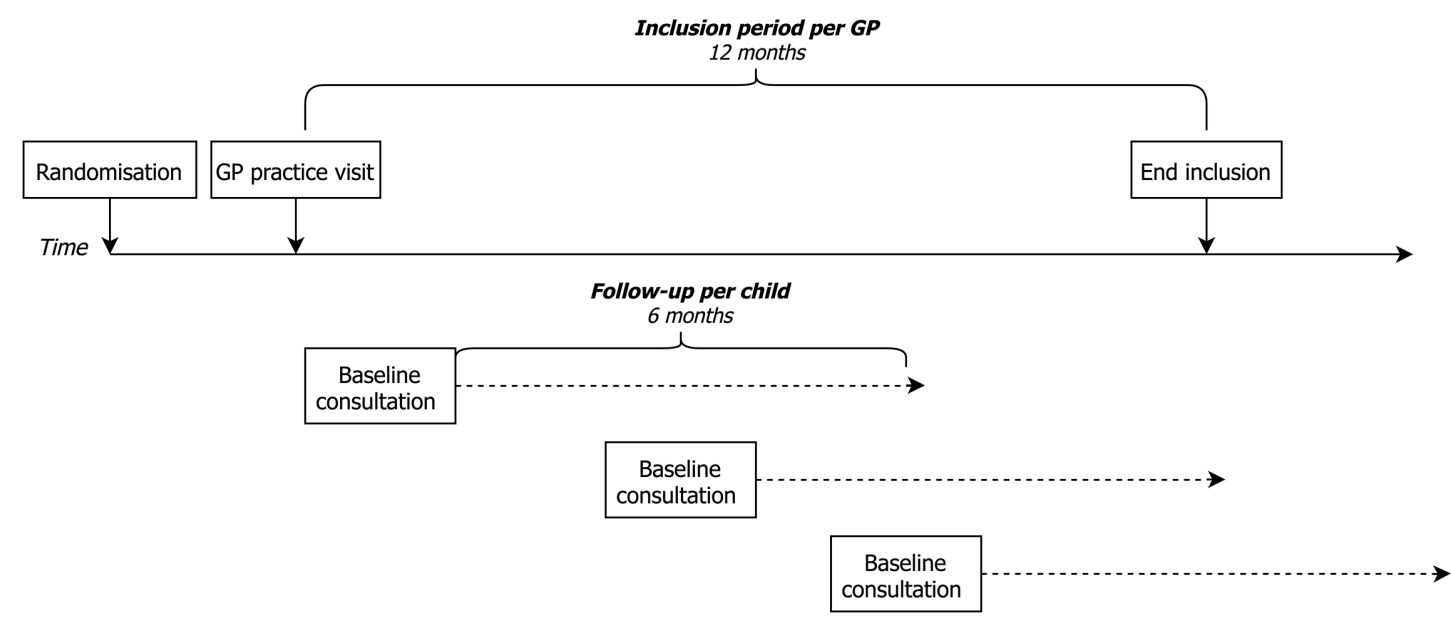

Figure 3 Study timeline at each GP practice. After a GP practice agrees to participate in the study, it is randomised to either the intervention or control group. Shortly thereafter, research staff visits the practice to explain study procedures, which marks the start of the 12 month inclusion period. GPs in the intervention group complete the online training before this visit. Children presenting with chronic gastrointestinal symptoms before the inclusion period starts are not eligible. Follow-up is 6 months for each child. GP, general practitioner.

receive reminders via e-mail after 7 and 14 days. If not completed after two reminders, we will call the child and/ or parents by phone.

\section{Sample size}

Based on our earlier study on the diagnostic value of FCal in primary care ${ }^{18}$ as well as the cross-sectional study on the management of children with abdominal pain in primary care, ${ }^{3}$ we expect referrals of children with chronic gastrointestinal symptoms to reduce from $17 \%$ to $7 \%$. To detect this difference with a power of $80 \%$ and a significance level of $5 \%$, an individually randomised study would need 326 children (163/arm). Given a mean cluster size (expected recruitment rate per practice) of 3 and an intraclass correlation coefficient of $0.06,{ }^{28}{ }^{46}$ we would need 366 children (183/arm). Then, allowing for a loss to follow-up of $10 \%$, this increases to 406 children (203/arm) from 134 general practices (67/arm). We assume that $15 \%$ of the practices will not recruit any children; therefore, we aim to recruit 158 general practices (79/arm).

\section{Analysis}

We will use descriptive statistics to summarise the data of GPs and children in the intervention and control groups, starting with their baseline characteristics. All analyses will be presented as estimates of intervention effects (adjusted mean differences or ORs, as appropriate), with associated $95 \%$ CIs and $\mathrm{p}$ values. Analysis for both the primary and secondary outcomes will initially be done on an intention-to-treat basis, with children analysed within the GP group in which they are registered, irrespective of the care received. Analyses will then be repeated for both the primary and secondary outcomes on a per protocol basis. In the intervention group, we will only include children who receive the intended diagnostic strategy (per the indications explained in the online training and with a returned stool sample) or rightfully did not receive
FCal testing (without alarm symptoms) and in the control group, we will only include children who did not undergo FCal testing. We will analyse the primary outcome by multilevel logistic regression modelling to account for the practice. The effect of the intervention on secondary parameters will be assessed by multilevel logistic (dichotomous variables) or linear (continuous variables) regression modelling, as appropriate.

\section{Economic evaluation}

Alongside the RCT, we will perform a cost-effectiveness study with two aims. The primary aim will be to study the incremental costs of FCal-POCT compared with care as usual from a societal perspective. If the new test strategy reduces the number of referrals, this will be visible as a cost reduction in the economic evaluation. An incremental cost-utility ratio will then be calculated, based on the EQ-5D-Y for assessing utility. The secondary aim will be to estimate the cost-effectiveness of FCal-POCT. Two incremental cost-effectiveness ratios will be calculated, using parental concern and parental satisfaction as effect parameters. Costs will be measured from a societal perspective, such that productivity losses incurred by parents will also be included. Healthcare consumption will be valued according to Dutch standard guidelines for economic evaluations. ${ }^{47}$ Bootstrap resampling will be performed on the costs (primary analysis) and on the cost-effect pairs (cost-effectiveness and cost-utility) to produce CIs. Finally, cost-effectiveness planes and acceptability curves will be plotted.

\section{Patient and public involvement}

We have collaborated with the Foundation Child and Hospital (Stichting Kind en Ziekenhuis) and have incorporated their opinions and expertise in the grant proposal, patient information letters and recruitment strategies. Moreover, we will ask them to help disseminate the study results to the public. In addition, we will distribute the 
study results to participating children and/or parents via a short e-mail newsletter. The Dutch Gastroenterology and Hepatology Foundation supports our research question and will also be involved in the dissemination of results.

\section{ETHICS AND DISSEMINATION}

\section{Ethics approval and consent to participate}

The Medical Research Ethics Committee (MREC) of the University Medical Center Groningen (Netherlands) (number: 201900309) approved this study. The ethics committee waived the requirement to obtain written informed consent for collecting data from patients' medical files, according to Dutch law (Medical Treatment Contracts Act). This was allowed because asking for written informed consent from children and/or their parents could jeopardise recruitment. Additionally, it will reduce the risk of selection bias and increase the generalisability of our results to a real-world setting. For the assessment of secondary outcomes by questionnaires, informed consent will be obtained either from parents alone (child $<12$ years), parents and child (child 12-15 years) or the child alone ( $>15$ years), consistent with Dutch law. Additionally, all participating GP practices will be required to sign a study agreement consenting to study protocol adherence and data collection by researchers from medical files. Important protocol changes will be communicated to the ethics committee and participating practices.

\section{Dissemination}

We aim to embed our study results in clinical practice. The findings will, therefore, be made available to patients, GPs, paediatricians and laboratories via presentations at national and international conferences, social media and peer-reviewed publications, irrespective of the magnitude or direction of effect. Within current national and international guidelines, there is a knowledge gap about the use of FCal in children in primary care. As such, our results will provide high-quality evidence according to Grading of Recommendations, Assessment, Development and Evaluations criteria because we include the impact on patient-important outcomes. ${ }^{48}$ The data of this study will be available on request.

\section{DISCUSSION}

To the best of our knowledge, this is the first trial designed to evaluate the effect of using FCal in the diagnostic process of GPs and how this affects referral rates for children with chronic gastrointestinal symptoms. We assume that children and/or parents in the intervention group will have improved patient-important outcomes due to the reduced diagnostic uncertainty. As such, we hypothesise that the referral rate will decrease. Although additional costs will be incurred by using FCal-POCT in the intervention group, we expect total costs to be lower compared with usual care because of the reduced use of other healthcare services (eg, fewer GP consultations, blood tests and referrals) as well as less productivity loss for parents.

There is increasing awareness that new medical tests should have scientifically proven patient benefits before they are implemented in healthcare guidelines. In 2014, Horvath et aldescribed a new cyclical framework for evaluating in vitro medical tests, and this consisted of analytical and clinical performance, clinical and cost-effectiveness and broader impact. ${ }^{49}$ The first steps in Horvath's framework, analytical and clinical performance, have already been evaluated for FCal-POCT. ${ }^{18}{ }^{25}$ In this trial, we will evaluate the impact of the test in daily practice, focusing on its clinical effectiveness and cost-effectiveness. An additional qualitative study is also needed to evaluate the broader impact of the FCal-POCT in primary care among GPs, GP assistants, parents and children. If our hypothesis is confirmed, we anticipate that there may be sufficient evidence to include a recommendation on the use of FCal-POCT in relevant guidelines for children with chronic gastrointestinal symptoms in primary care.

Our choice of a clustered trial design may raise some questions. We chose this approach because it is not feasible to randomise the intervention at an individual level since it would be very demanding for GPs to change their diagnostic strategy for each child. Additionally, it is not desirable to randomise at a GP level due to the risk of contamination between GPs working in the same practice. ${ }^{50}$ Nevertheless, we concede that the clustered randomised trial design has some limitations. ${ }^{51-53}$ First, blinding the participating GPs is neither feasible nor desirable because the transfer from care as usual to intervention is obvious. To reduce bias, those who perform the analysis will be blinded to the assigned study group. Second, the cluster effect must be considered ${ }^{46}$ given that participants within one cluster may share certain characteristics (eg, quality of care at the GP practice) that could substantially affect power. Therefore, we corrected for the cluster effect in the sample size calculation by using an intraclass correlation coefficient of 0.06 , which is higher than used in most cluster trials in primary care. ${ }^{46} 54$ Finally, this trial design is prone to selection bias, ${ }^{51-53} 55$ with GPs in the intervention group potentially including participants with different characteristics to those in the control group due to the knowledge gained (eg, alarm symptoms) in the online training. Although research staff will search for eligible children in the GPs' registration databases to reduce this risk, it should be noted that this process may be prone to the same bias. ${ }^{56}$

When designing this study, we used the PRagmatic Explanatory Continuum Indicator Summary (PRECIS)-2 tool to match our design to the intended purpose: a pragmatic yet valid trial. ${ }^{57}$ We opted for a pragmatic design, so that we could reflect the effectiveness of the intervention in routine clinical practice ${ }^{57}$ Such trials are also highly generalisable and produce externally valid results that are relevant to decision-makers. ${ }^{5-60}$ However, unlike in explanatory trials, protocol adherence is rarely monitored and the degree to 
which the intervention is implemented in daily clinical practice often remains uncertain. ${ }^{61}$ Therefore, any real effect could be masked by a large amount of variation. ${ }^{62}$ This will be addressed by monitoring whether GPs comply with the protocol and by performing a per-protocol analysis.

This study evaluates the impact of a test strategy in which FCal testing is a major component. It will be impossible to distinguish whether a possible effect can be attributed to FCal testing or to the training. However, we think that these two elements should go hand-in-hand in order to increase the compliance to the new test strategy and prevent missed diagnoses, over-diagnosis and unnecessary costs for patients and wider society. ${ }^{22}{ }^{63}$ This is of similar importance when a test is implemented in a real-world setting.

In conclusion, we seek to evaluate the effect of an FCalPOCT test strategy in children with chronic gastrointestinal symptoms in primary care. If the intervention is shown to be clinically beneficial and cost effective, we will be able to promote its uptake in everyday practice, where we expect it to have a positive impact on children presenting with chronic gastrointestinal symptoms in primary care.

Correction notice This article has been corrected since it was published. Author name 'Patrick Ferry van Rheenen' has been updated.

Twitter Patrick Ferry van Rheenen @PFvRheenen

Acknowledgements We are very grateful to J. Pruijsen (Department of Paediatric Gastroenterology, University Medical Center Groningen, the Netherlands), A. Heerdink (Department of Medical Sciences, University Medical Center Groningen, the Netherlands), H. Raghoebar-Krieger (Department of General Practice, University Medical Center Groningen, the Netherlands), A. Diemers (Department of General Practice and Wenckebach Institute for Education and Training, University Medical Center Groningen, the Netherlands) and A. Muller Kobold (Department of Laboratory Medicine, University Medical Center Groningen, The Netherlands) for helping us develop the online training. We want to thank Foundation Child and Hospital (Stichting Kind en Ziekenhuis) for helping us with the grant proposal, patient information letters and recruitment strategies. Additionally, we thank M. Wolf and $\mathrm{E}$. Hogervorst for helping us to collect and manage the data during the trial. Finally, we thank Dr Robert Sykes (www.doctored.org.uk) for providing editorial services.

Contributors GAH, PvR, KV and MYB conceived the original research concept. All authors contributed to the study design. SMA, MC and GB will collect and manage data during the trial. SMA has written and revised this protocol. All authors have contributed important intellectual content to the manuscript and have approved the final version for publication in this journal.

Funding This trial was supported by ZonMW, The Dutch Organization for Health Research and Development (project number 852001930). The BÜHLMANN Group funded testing. However, the funders had no role in the protocol development and will have no role in data collection, analysis, decision to publish or manuscript preparation. All authors will have full access to all data.

Competing interests None declared.

Patient consent for publication Not required.

Provenance and peer review Not commissioned; externally peer reviewed.

Supplemental material This content has been supplied by the author(s). It has not been vetted by BMJ Publishing Group Limited (BMJ) and may not have been peer-reviewed. Any opinions or recommendations discussed are solely those of the author(s) and are not endorsed by BMJ. BMJ disclaims all liability and responsibility arising from any reliance placed on the content. Where the content includes any translated material, BMJ does not warrant the accuracy and reliability of the translations (including but not limited to local regulations, clinical guidelines, terminology, drug names and drug dosages), and is not responsible for any error and/or omissions arising from translation and adaptation or otherwise.

Open access This is an open access article distributed in accordance with the Creative Commons Attribution Non Commercial (CC BY-NC 4.0) license, which permits others to distribute, remix, adapt, build upon this work non-commercially, and license their derivative works on different terms, provided the original work is properly cited, appropriate credit is given, any changes made indicated, and the use is non-commercial. See: http://creativecommons.org/licenses/by-nc/4.0/.

ORCID iDs

Sophie Ansems http://orcid.org/0000-0002-8744-8224

Gea Holtman http://orcid.org/0000-0001-6579-767X

\section{REFERENCES}

1 Gieteling MJ, Lisman-van Leeuwen Y, van der Wouden JC, et al. Childhood nonspecific abdominal pain in family practice: incidence, associated factors, and management. Ann Fam Med 2011;9:337-43.

2 Spee LAA, van den Hurk APJM, van Leeuwen Y, et al. Childhood abdominal pain in primary care: design and patient selection of the HONEUR abdominal pain cohort. BMC Fam Pract 2010;11:27.

3 Spee LAA, Lisman-Van Leeuwen Y, Benninga MA, et al. Prevalence, characteristics, and management of childhood functional abdominal pain in general practice. Scand J Prim Health Care 2013;31:197-202.

4 Hyams JS, Di Lorenzo C, Saps M, et al. Childhood functional gastrointestinal disorders: Child/Adolescent. Gastroenterology 2016;150:1456-68.

5 Gieteling MJ, Bierma-Zeinstra SMA, Passchier J, et al. Prognosis of chronic or recurrent abdominal pain in children. J Pediatr Gastroenterol Nutr 2008;47:316-26.

6 van Tilburg MAL, Venepalli N, Ulshen M, et al. Parents' worries about recurrent abdominal pain in children. Gastroenterol Nurs 2006;29:50-5.

7 Sawczenko A, Ballinger AB, Savage MO, et al. Clinical features affecting final adult height in patients with pediatric-onset Crohn's disease. Pediatrics 2006;118:124-9.

8 Walters TD, Kim M-O, Denson LA, et al. Increased effectiveness of early therapy with anti-tumor necrosis factor- $\alpha$ vs an immunomodulator in children with Crohn's disease. Gastroenterology 2014;146:383-91.

9 Laurikka P, Nurminen S, Kivelä L, et al. Extraintestinal manifestations of celiac disease: early detection for better long-term outcomes. Nutrients 2018;10. doi:10.3390/nu10081015. [Epub ahead of print: 03 Aug 2018].

10 Ricciuto A, Mack DR, Huynh HQ, et al. Diagnostic delay is associated with complicated disease and growth impairment in paediatric Crohn's disease. J Crohns Colitis 2021;15:419-31.

11 Adler J, Lin CC, Gadepalli SK, et al. Association between steroidsparing therapy and the risk of perianal Fistulizing complications among young patients with Crohn disease. JAMA Netw Open 2020;3:e207378.

12 Kugathasan S, Denson LA, Walters TD, et al. Prediction of complicated disease course for children newly diagnosed with Crohn's disease: a multicentre inception cohort study. Lancet 2017;389:1710-8.

13 Gieteling MJ, van Dijk PA, de Jonge AH. NHG-Standaard Buikpijn bij kinderen. Huisarts Wet 2012;55:404-9.

14 Holtman GA, Lisman-van Leeuwen Y, Kollen BJ, et al. Diagnostic test strategies in children at increased risk of inflammatory bowel disease in primary care. PLoS One 2017;12:e0189111.

15 Holtman GA, Lisman-van Leeuwen Y, Reitsma JB, et al. Noninvasive tests for inflammatory bowel disease: a meta-analysis. Pediatrics 2016;137:e20152126.

16 Mack DR, Langton C, Markowitz J, et al. Laboratory values for children with newly diagnosed inflammatory bowel disease. Pediatrics 2007;119:1113-9.

17 Kazak AE, Kassam-Adams N, Schneider S, et al. An integrative model of pediatric medical traumatic stress. J Pediatr Psychol 2006;31:343-55.

18 Holtman GA, Lisman-van Leeuwen Y, Kollen BJ, et al. Diagnostic accuracy of fecal calprotectin for pediatric inflammatory bowel disease in primary care: a prospective cohort study. Ann Fam Med 2016;14:437-45.

19 Ramraj R, Garcia A, Mosen D, et al. Utility of fecal calprotectin in evaluation of chronic gastrointestinal symptoms in primary care. Clin Pediatr 2018;57:1058-63.

20 Walker GJ, Chanchlani N, Thomas A, et al. Primary care faecal calprotectin testing in children with suspected inflammatory bowel disease: a diagnostic accuracy study. Arch Dis Child 2020;105:957-63.

21 Ludvigsson JF, Büsch K, Olén O, et al. Prevalence of paediatric inflammatory bowel disease in Sweden: a nationwide populationbased register study. BMC Gastroenterol 2017;17:23. 
22 Schols AM, Dinant G-J, Cals JW. Point-Of-Care testing in general practice: just what the doctor ordered? Br J Gen Pract 2018;68:362-3.

23 Howick J, Cals JWL, Jones C. Current and future use of pointof-care tests in primary care: an international survey in Australia, Belgium, the Netherlands, the UK and the USA. BMJ Open 2014;4:e005611.

24 Turner PJ, Van den Bruel A, Jones CHD, et al. Point-Of-Care testing in UK primary care: a survey to establish clinical needs. Fam Pract 2016;33:388-94.

25 Holtman GA, Lisman-van Leeuwen Y, van Rheenen PF, et al. Evaluation of point-of-care test calprotectin and lactoferrin for inflammatory bowel disease among children with chronic gastrointestinal symptoms. Fam Pract 2016;154:cmw079.

26 Heida A, Knol M, Kobold AM, et al. Agreement between home-based measurement of stool calprotectin and ELISA results for monitoring inflammatory bowel disease activity. Clin Gastroenterol Hepatol 2017:15:1742-9.

27 Haisma S-M, Galaurchi A, Almahwzi S, et al. Head-To-Head comparison of three stool calprotectin tests for home use. PLoS One 2019;14:e0214751.

28 Cals JWL, Hopstaken RM, Butler CC, et al. Improving management of patients with acute cough by C-reactive protein point of care testing and communication training (IMPAC3T): study protocol of a cluster randomised controlled trial. BMC Fam Pract 2007;8:15.

29 Chan A-W, Tetzlaff JM, Altman DG, et al. Spirit 2013 statement: defining standard protocol items for clinical trials. Ann Intern Med 2013;158:200-7.

30 Campbell MK, Piaggio G, Elbourne DR, et al. Consort 2010 statement: extension to cluster randomised trials. BMJ 2012;345:e5661.

31 Lucassen P, Albeda F, Van Reisen M. NHG-Standaard Voedselovergevoeligheid | NHG. Huisarts Wet 2010;53:537-53.

32 Bruhl P, Lamers H, Van Dongen A, et al. NHG-Standaard acute diarree |NHG. Huisarts Wet 2007;3:103-13.

33 Diemel J, Van den Hurk A, Muris J, et al. NHG-Standaard Obstipatie NHG. Huisarts Wet 2010;53:484-98.

34 BÜHLMANN Laboratories AG. Intended use. In: IBDoc manual. version 4. Schönenbuch. 3. In, 2019.

35 Kirkpatrick DL. Evaluation of Training. In: Training and development Handbook. New York: McGraw Hillbook Company, 1967: 40-60.

36 Roca M, Rodriguez Varela A, Carvajal E, et al. Fecal calprotectin in healthy children aged 4-16 years. Sci Rep 2020;10.

37 Hopstaken RM, van Balen JAM, Kusters R. [Point-of-care-testing in general practice]. Ned Tijdschr Geneeskd 2015;159:A9475.

38 Lewis CC, Scott DE, Pantell RH, et al. Parent satisfaction with children's medical care. development, field test, and validation of a questionnaire. Med Care 1986;24:209-15.

39 Hart CN, Drotar D, Gori A, et al. Enhancing parent-provider communication in ambulatory pediatric practice. Patient Educ Couns 2006;63:38-46.

40 Lemiengre MB, Verbakel JY, De Burghgraeve T, et al. Optimizing antibiotic prescribing for acutely ill children in primary care (ERNIE2 study protocol, part B): a cluster randomized, factorial controlled trial evaluating the effect of a point-of-care C-reactive protein test and a brief intervention combined with written safety net advice. BMC Pediatr 2014;14:1-9.

41 Baber KF, Anderson J, Puzanovova M, et al. Rome II versus Rome III classification of functional gastrointestinal disorders in pediatric chronic abdominal pain. J Pediatr Gastroenterol Nutr 2008:47:299-302.

42 Claar RL, Walker LS. Functional assessment of pediatric pain patients: psychometric properties of the functional disability inventory. Pain 2006;121:77-84.
43 Wille N, Badia X, Bonsel G, et al. Development of the EQ5D-Y: a child-friendly version of the EQ-5D. Qual Life Res 2010;19:875-86.

44 Ravens-Sieberer U, Wille N, Badia X, et al. Feasibility, reliability, and validity of the EQ-5D-Y: results from a multinational study. Qual Life Res 2010;19:887-97.

45 Bouwmans C, Hakkaart-van Roijen L. Handleiding iMTA productivity cost questionnaire (iPCQ). Rotterdam: iMTA, Erasmus Universiteit, 2013.

46 Rutterford C, Copas A, Eldridge S. Methods for sample size determination in cluster randomized trials. Int $\mathrm{J}$ Epidemiol 2015;44:1051-67.

47 Hakkaart-van Roijen L, Van der Linden N, Bouwmans C. Methodologie van kostenonderzoek en referentieprijzen voor economische evaluaties in de gezondheidszorg.In opdracht van Zorginstituut Nederland. Geactualiseerde versie 2015.

48 Schünemann HJ, Schünemann AHJ, Oxman AD, et al. Grading quality of evidence and strength of recommendations for diagnostic tests and strategies. BMJ 2008;336:1106-10.

49 Horvath AR, Lord SJ, StJohn A, et al. From biomarkers to medical tests: the changing landscape of test evaluation. Clin Chim Acta 2014;427:49-57.

50 Teerenstra S, Melis RJF, Peer PGM, et al. Pseudo cluster randomization dealt with selection bias and contamination in clinical trials. J Clin Epidemiol 2006;59:381-6.

51 Hahn S, Puffer S, Torgerson DJ, et al. Methodological bias in cluster randomised trials. BMC Med Res Methodol 2005;5:10.

52 Puffer S, Torgerson D, Watson J. Evidence for risk of bias in cluster randomised trials: review of recent trials published in three general medical journals. BMJ 2003;327:785-9.

53 Brierley G, Brabyn S, Torgerson D, et al. Bias in recruitment to cluster randomized trials: a review of recent publications. J Eval Clin Pract 2012;18:878-86.

54 Adams G, Gulliford MC, Ukoumunne OC, et al. Patterns of intracluster correlation from primary care research to inform study design and analysis. J Clin Epidemiol 2004;57:785-94.

55 Bolzern J, Mnyama N, Bosanquet K, et al. A review of cluster randomized trials found statistical evidence of selection bias. J Clin Epidemiol 2018;99:106-12.

56 Eldridge S, Kerry S, Torgerson DJ. Bias in identifying and recruiting participants in cluster randomised trials: what can be done? BMJ 2009;339:b4006-9.

57 Loudon K, Treweek S, Sullivan F, et al. The PRECIS-2 tool: designing trials that are fit for purpose. BMJ 2015;350:h2147.

58 Singal AG, Higgins PDR, Waljee AK. A primer on effectiveness and efficacy trials. Clin Trans/ Gastroenterol 2014;5:e45.

59 Patsopoulos NA. A pragmatic view on pragmatic trials. Dialogues Clin Neurosci 2011;13:217-24.

60 Eldridge S. Pragmatic trials in primary health care: what, when and how? Fam Pract 2010;27:591-2.

61 Laxy M, Wilson ECF, Boothby CE, et al. How good are GPs at adhering to a pragmatic trial protocol in primary care? results from the ADDITION-Cambridge cluster-randomised pragmatic trial. BMJ Open 2018;8:e015295

62 Forbes G, Loudon K, Clinch M, et al. Improving the relevance of randomised trials to primary care: a qualitative study investigating views towards pragmatic trials and the PRECIS-2 tool. Trials 2019;20:711.

63 André M, Schwan A, Odenholt I, et al. The use of CRP tests in patients with respiratory tract infections in primary care in Sweden can be questioned. Scand J Infect Dis 2004;36:192-7. 